\title{
PERIODIC STRUCTURES MANUFACTURED BY 3D PRINTING FOR ELECTRON BEAM EXCITATION OF HIGH POWER MICROWAVE SOURCES*
}

\author{
A.R. Phippss ${ }^{1}$ A.J. MacLachlan ${ }^{1}$, L. Zhang ${ }^{1}$, C.R. Robertson ${ }^{1}$, I.V. Konoplev ${ }^{2}$, K. Ronald ${ }^{1}$, \\ A.D.R. Phelps ${ }^{1}$ and A.W. Cross ${ }^{1}$ \\ ${ }^{1}$ Department of Physics, SUPA, University of Strathclyde, 107 Rottenrow, Glasgow, G4 ONG, Scotland, UK \\ ${ }^{3}$ The John Adams Institute, Department of Physics, University of Oxford, Oxford OX1 3RH, UK.
}

\section{Abstract}

A Two-dimensional (2D) Periodic Surface Lattice (PSL) can produce distributed feedback in high power microwave sources driven by an oversized electron beam of annular geometry. Such 2D periodic structures can be formed in cylindrical waveguide with a grating machined on the walls, where the diameter of the waveguide is larger than the wavelength $\lambda(\mathrm{D}>>\lambda)$. In this case localised surface fields can be excited around the perturbations if the structure is radiated by an external source (for example an electron beam). Experiments were conducted using a velvet cathode electron gun with the electron accelerating voltage produced by a cable Blumlein generator. "Additive Manufacturing" or "3D printing" was used to construct a silver 2D PSL quickly, efficiently and relatively inexpensively. The electron beam formed within an $18 \mathrm{~mm}$ bore $1.8 \mathrm{~T}$ solenoid was transported through the $7.2 \mathrm{~mm}$ inner diameter silver 2D PSL beam-wave interaction region. An $80 \mathrm{kV}, 100 \mathrm{~A}$ electron beam with an outer diameter of $4 \mathrm{~mm}$ and inner diameter of $2 \mathrm{~mm}$ which was approximately $1.8 \mathrm{~mm}$ away from the inner surface of the 2D PSL corrugation was measured. Millimetre wave radiation at a frequency of $\sim 80 \mathrm{GHz}$ at an output power of $130 \pm 30 \mathrm{~kW}$ corresponding to an operating efficiency of $\sim 1.7 \%$ was measured.

\section{INTRODUCTION}

To increase the power available to a beam wave interaction either the energy of the beam may be increased or the current flowing in the beam [1-3]. Electron beam density cannot be arbitrarily increased however as at some point the internal space charge forces on the beam, and the influence of the surrounding environment contrive to impose limits on the beam stability and propagation. In addition, the increased RF power density can result in breakdown and the effect known as pulse shortening. To avoid these issues, it is necessary to consider the possibility of increasing the transverse size of the beam and therefore, in general, of the microwave circuit itself, if a further increase of power is to be achieved [4-6]. Unfortunately, increasing the transverse size of the microwave circuit beyond a ratio of $D / \lambda \sim 4$ (where $D$ is the transverse size of the microwave circuit and $\lambda$ is the radiation wavelength) results in a drop in efficiency and spectral purity as the source loses mode selectivity [7-9]. To tackle this problem, the use of a $2 \mathrm{D}$ periodic surface lattice resulting in the coupling of 4 waves (a forward and backward longitudinal wave and a clockwise and anticlockwise azimuthal wave) to control the transverse modes which can interact with the electron beam in an oversized interaction space has been proposed [4]. This enables high efficiency even when the transverse size of the electron beam exceeds the radiation wavelength by more than 2 to 3 orders of magnitude. Additive Manufacturing [10] or "3D printing" was used to construct a silver alloy $2 \mathrm{D}$ periodic surface lattice structure that was designed to operate as a $\mathrm{W}$-band source (75-110GHz).

\section{II.RESULTS}

\section{A. 3D printed 2D Periodic Surface Lattice}

3D printing developed in the mid 1980's [10] and the number of its applications is continuing to expand. The tolerances achievable with $3 \mathrm{D}$ printing are projected to improve over time as the technology develops, resulting in higher degrees of accuracy [11]. 3D printing is used in the initial creative process that results in structures such as the example shown in Figure 1. It involves an additive process by which consecutive layers in the $\mathrm{x}-\mathrm{z}$ plane are deposited sequentially in the +ve y direction. The printing process follows the pattern in a given CAD input file, usually in the STL (Stereolithograph) file format where every face is built from a series of interconnected triangles represented by 3 separate 32- bit floating-point Cartesian coordinates. The physical parameters of the $\mathrm{W}$ -

\footnotetext{
* Work supported in part by the EPSRC who sponsored the research studentships of A.R. Phipps and A.J. MacLachlan. The authors acknowledge the support of the Leverhulme Trust Network "Advanced Research on Generation of THz and X-ray Radiation" (IN-2015-012). This material is based upon work supported by the Air Force Office of Scientific Research under award numbers FA8555-13-1-2132 and FA9550-17-1-0095.
} 
band $(75-110 \mathrm{GHz}) 2 \mathrm{D}$ PSL are shown in Table 1. The design is sliced into digital layers so that a curve is 'approximated' by many square-sided slices, with the thickness of each layer representing the resolution of that particular 3D printing process.

- The cylindrical 2D periodic structure can be described by equation (1)

$$
r=r_{0}+\Delta r \cos \left(k_{z} z+m \varphi\right)
$$

where $\mathrm{r}_{0}$ is the radius of the unperturbed waveguide, $\Delta \mathrm{r}$ is the amplitude of the corrugation, $k_{\mathrm{z}}=2 \pi / d_{z}$, where $d_{z}$ is the period of the corrugation over the $\mathrm{z}$ - coordinate and $m$ is the number of azimuthal variations of the corrugation

Table 1. The physical parameters of the 2D PSL.

\begin{tabular}{|l|c|c|}
\hline Symbol & Quantity & Dimensions \\
\hline $\mathrm{dz}$ & longitudinal period & $1.6 \mathrm{~mm}$ \\
\hline $\mathrm{m}$ & azimuthal variations & 7 \\
\hline $\mathrm{N}$ & $\begin{array}{c}\text { number of longitudinal } \\
\text { periods }\end{array}$ & 16 \\
\hline$\Delta \mathrm{r}$ & perturbation amplitude & $0.8 \mathrm{~mm}$ \\
\hline$r_{\min }$ & $\begin{array}{c}\text { minimum radius of } \\
\text { perturbation }\end{array}$ & $3.6 \mathrm{~mm}$ \\
\hline$r_{\max }$ & $\begin{array}{c}\text { maximum radius of } \\
\text { perturbation }\end{array}$ & $5.2 \mathrm{~mm}$ \\
\hline
\end{tabular}

The perturbation amplitude is 800 microns $(1.6 \mathrm{~mm}$ peak to peak). Representation of this structure with layers of 16 microns results in a reasonable level of accuracy and the finished component appears to hold an adequate level of detail. In the case of this specific piece the complete part was printed in wax and this former was then used to create a suitable mold into which a molten silver (92.5\%) chromium $(7.5 \%)$ alloy was set, with the resultant component having an overall final resolution within \pm 125 microns

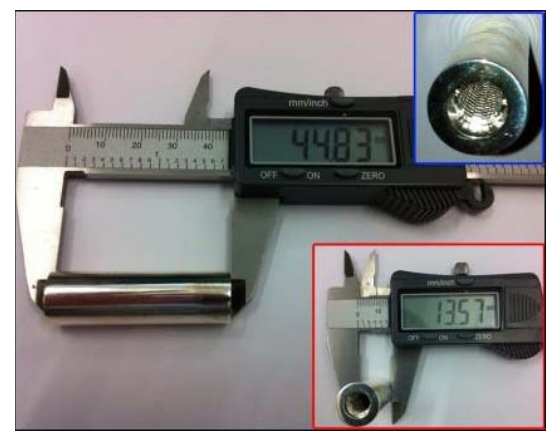

Figure 1 Cylindrical PSL manufactured using 3D printing

The 2D PSL structure was successfully incorporated in a cold test system with the transmission of millimeter waves measured using a Vector Network Analyzer (VNA). When testing the structure using the VNA the interaction uses an electromagnetic wave that interacts with the $1.6 \mathrm{~mm}$ longitudinal period to give a response in G-band, resulting in a resonance at $187.7 \mathrm{GHz}$, as shown in Fig. 2. The electromagnetic wave when interacting with the $3.5 \mathrm{~mm}$ azimuthal period gives a fundamental resonance in $\mathrm{W}$-band at $\sim 85 \mathrm{GHz}$ and a resonance at the 1st harmonic at $171.1 \mathrm{GHz}$ in G-band. This is what gives the $171.1 \mathrm{GHz}$ resonance shown in the VNA measurement.

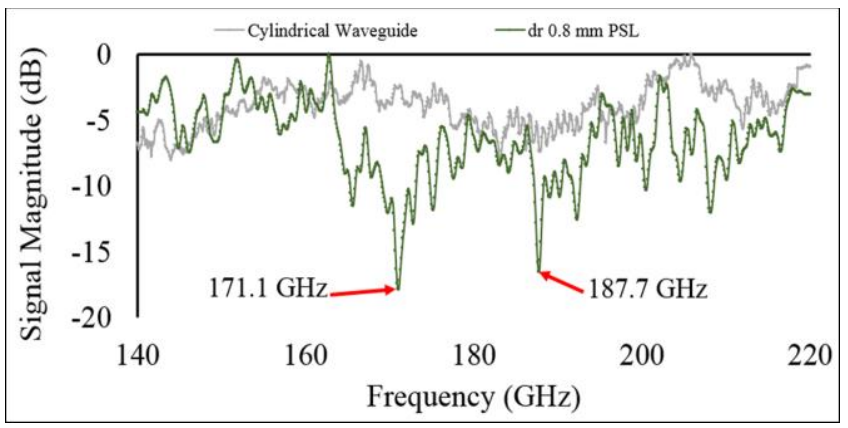

Figure 2 The measured transmission as a function of frequency of the 2D PSL over the $140 \mathrm{GHz}$ to $220 \mathrm{GHz}$ frequency range.

The response of the structure has been measured in the G-band (140-220 GHz) to test the performance of the structure and to verify that both the longitudinal and azimuthal periodicities have been correctly manufactured. The electron beam driven 2D PSL is a W-band structure from the point of view of the frequency of the output of the radiation source. It is because the electron beam is travelling in this case at about half the speed of light and it is the electron beam when operating as a source that is exciting the longitudinal structure in W-band. The corresponding fundamental azimuthal resonant electromagnetic response, as noted above, is in W-band and is designed to correspond with the electron beam excitation of the longitudinal structure in W-band.

Following VNA characterization measurements the 3D printed 2D PSL structure was incorporated into a high power millimeter wave source.

\section{B Electron Beam Experiments}

The electron gun was operated in the space-charge limited regime of electron field emission, using an annular velvet disk to enhance the electron emission properties of the cathode. A stacked double-Blumlein pulsed high voltage source was used to provide the accelerating field [12]. As shown in figure 3 for a beam acceleration potential of $\sim 80 \mathrm{kV}$, a beam current of $100 \mathrm{~A}$ was measured.

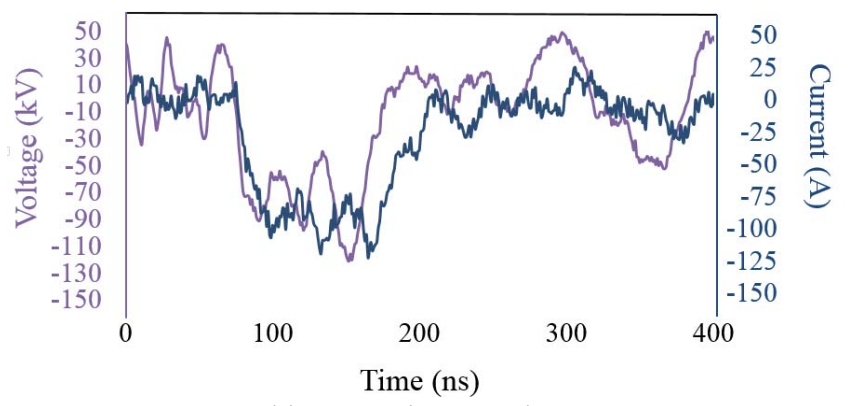

Figure 3 Measured beam voltage and current 
For accurate evaluation of interaction characteristics, the beam current was measured in the 2D PSL cavity using an in-line Faraday cup to collect the electrons with a $10 \Omega$ current shunt used with the voltage further reduced using $30 \mathrm{~dB}$ of attenuation and then measured on a digital storage oscilloscope. The Faraday cup beam diagnostic was later removed when the high power millimeter wave experiments were conducted. The electron beam was formed, focused and transported through the 2D PSL interaction region using a 1.81 Tesla continuous water cooled solenoid of length $30 \mathrm{~cm}$ and inner diameter of $14 \mathrm{~mm}$ as shown in figure 4

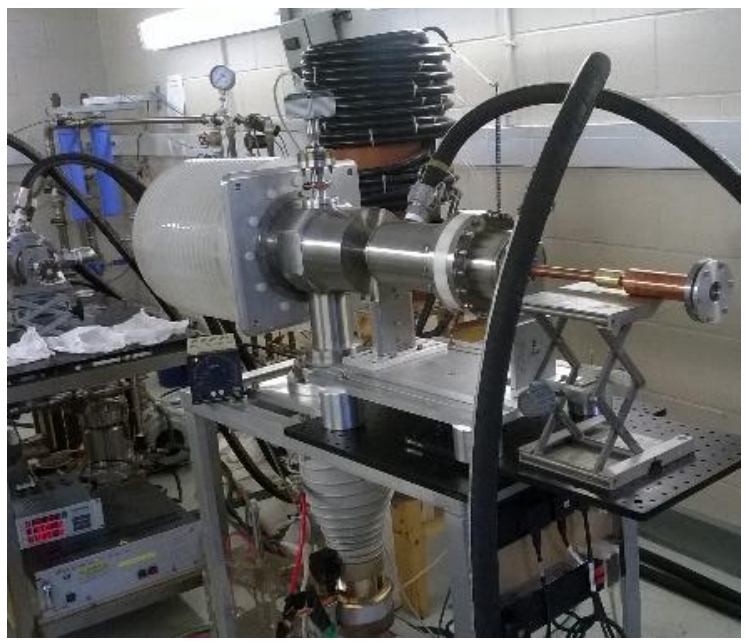

Figure 4 Photograph of 2D PSL high power mm-wave source showing the Blumlein cable pulser, diode, water cooled solenoid, output horn and window and diffstack pumping system.

The transverse profile of the electron beam was measured using electron sensitive film fixed on the front face of the Faraday cup and placed at the end of the 2D PSL interaction region. A typical electron beam transverse pulse profile measured by the witness plate is shown in figure 5. For one pulse at a cavity B-field of $1.81 \mathrm{~T}$ a slightly off centre annular electron beam was observed as shown in the image of the witness plate, figure 5.

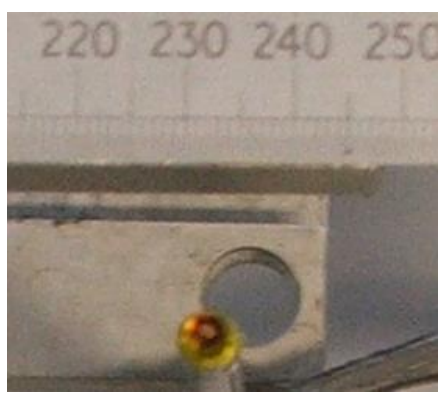

Figure 5 The electron sensitive witness plate shows the shape of the electron beam after one pulse has passed through the 2D PSL interaction region.

The yellow witness plate film turns red when high energy electrons hit it. It can be clearly seen on the $6 \mathrm{~mm}$ diameter witness plate that the electron beam is displaced slightly from the center and has a annular shape with an outer diameter $4 \mathrm{~mm}$ and an inner diameter of $2 \mathrm{~mm}$.

\section{Millimeter Wave Measurements}

To measure the amplitude of the output radiation field from the 2D PSL experiment, a W-band (75 to $110 \mathrm{GHz}$ ) Flann rectifying crystal detector with a rotary vane $\mathrm{W}$ band attenuator and Flann $15 \mathrm{~dB}$ horn were used located at a distance of $0.6 \mathrm{~m}$ from the output window. The millimeter wave detector consisted of a rectangular horn to collect the generated millimeter wave signal, an attenuator to reduce the strength of the signal, a crystal detector to convert the millimeter wave signal into a voltage signal. The millimeter wave signal in $\mathrm{mVs}$ was recorded using a digital storage oscilloscope after passing through a series of high pass cut-off filters. When there was no cut-off filter, with only the $75 \mathrm{GHz}$ to $110 \mathrm{GHz}$ W-band rectangular waveguide section present the cut-off frequency of the detection system was measured to be 60 GHz. Figure 6 displays the power output as measured in $\mathrm{mV}$ from the crystal detector when a particular cut - off filter was in place in the detection system. A number of shots were taken for each filter with figure 6 representative of the signal detected after successive shots.

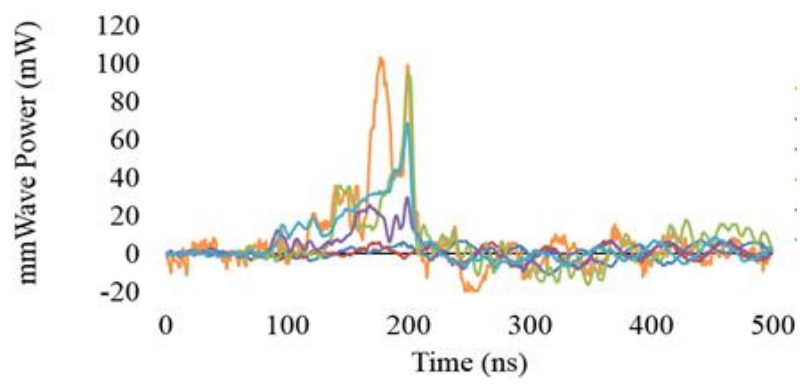

Figure 6 The power produced in the presence of a particular cut - off filter. Light blue line $70 \mathrm{GHz}$, Green line $-75 \mathrm{GHz}$, Purple line $80 \mathrm{GHz}$, red line $85 \mathrm{GHz}$ dark blue line $95 \mathrm{GHz}$. It is noted that using a $85 \mathrm{GHz}$ cut off filter results in no $\mathrm{mm}$ wave output at all with much reduced output measured for the cut-off filter above 80 $\mathrm{GHz}$ indicating that the signal lies somewhere between 80 $\mathrm{GHz}$ to $85 \mathrm{GHz}$.

Figure 6 demonstrates that the millimeter wave output lies between $80 \mathrm{GHz}$ and $85 \mathrm{GHz}$. Although the magnitude of the power varies a little when different filters were used it is clear that the mm wave output was produced at a frequency of interaction of the 2D PSL at $\sim 80 \mathrm{GHz}$.

In order to understand the mode generated, a farfield analysis was performed on the output mode launched through the horn and Mylar window. Here, a sector plate marked in single degree intervals, $\theta$, was used, with the output horn and window located at the centre of the semicircular plate and a rectangular waveguide receiving 
antenna mounted above the plate at the other end of the semi-circle. The apertures of the 2D PSL output horn and the detector receiving horn were separated by a distance, $0.6 \mathrm{~m}$. The receiving antenna was positioned on a moveable rail, allowing measurements that could be taken at values of $\theta$ between $-90^{\circ}$ and $90^{\circ}$. In order to ensure accuracy with the measurements, the output aperture of the experiment was placed directly above the pivoting point of the semi-circular plate. The mode scan results over one half of the scan with 0 representing the centre line is shown in figure 7.

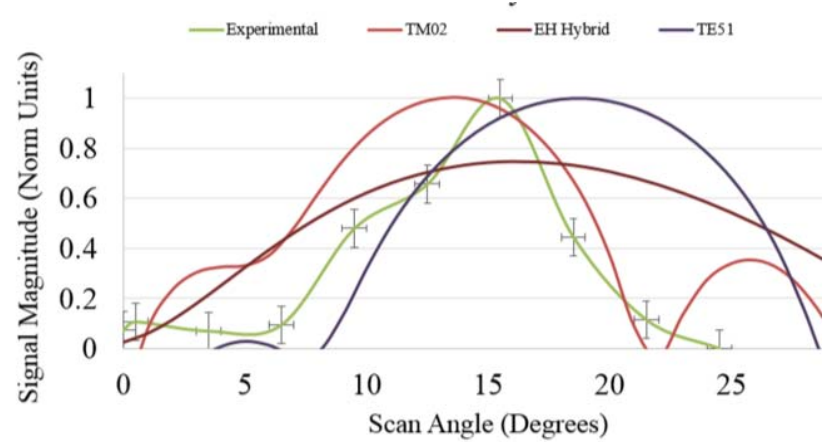

Figure 7 Green line represents the measured mode pattern. From the mode scan results measurements the peak of the green curve corresponds best to a combination of the $\mathrm{TM}_{0,2}$ mode and the $\mathrm{TE}_{5,1}$ mode

Although it is difficult to make a perfect match the mode pattern measurements indicates that the most likely mode is a combination of the $\mathrm{TM}_{0,2}$ volume and $\mathrm{TE}_{5,1}$ surface mode.

The output power was measured using the general antenna theorem with the total power from a launching antenna, calculated by integrating its radiated power density over space. The integration was completed by numerically integrating the normalized mode profile of the launching horn and multiplying by the measured maximum power density. After optimization of the experiment by adjustment of the location of the cathode a total power of the $\sim 80 \mathrm{GHz}$ source was found to be $130 \pm$ $30 \mathrm{~kW}$ resulting in a beam - wave interaction of $\sim 1.7 \%$ efficiency

\section{III.SUMMARY}

The mode pattern and cut-off filter measurements gives evidence of an interaction of a cavity eigenmode produced by the coupling of a TM 0,2 volume field and a $\mathrm{TE}_{5,1}$ surface field coupled within the 2D structure. However a more accurate frequency measurement using a heterodyne frequency diagnostic is required to conclusively prove that the coupling between the surface and volume field is the dominant beam-wave interaction in the experiment. A heterodyne frequency diagnostic experiment is planned as part of the future work.

\section{REFERENCES}

[1] A. W. Cross, I. V. Konoplev, K. Ronald, et al., "Experimental studies of two-dimensional coaxial Bragg structures for a high-power free-electron maser," Appl. Phys. Lett., vol. 80, pp. 1517-1519, March 2002.

[2] N.S. Ginzburg, N.Y. Peskov, A.S. Sergeev, et al., "Theory of free-electron maser with two-dimensional distributed feedback driven by an annular electron beam," J. Appl. Phys., vol. 92, pp. 1619-1629, Aug. 2002..

[3] N.S. Ginzburg, N.Y. Peskov, A.S. Sergeev, et al., "The use of a hybrid resonator consisting of one-dimensional and two-dimensional Bragg reflectors for generation of spatially coherent radiation in a coaxial free-electron laser,” Phys. Plasmas, vol. 9, pp. 2798-2802, June 2002.

[4] I. V. Konoplev, P. McGrane, W. He, A. W. Cross, A. D. R. Phelps, C. G. Whyte, K. Ronald, C. W. Robertson, "Experimental study of coaxial free-electron maser based on two-dimensional distributed feedback", Phys. Rev. Lett., vol.96, art. no. 035002, Jan. 2006.

[5] A.W. Cross, I.V. Konoplev, A.D.R. Phelps, et al., "Studies of surface two-dimensional photonic band-gap structures", J. Appl. Phys., vol. 93 pp. 2208-2218, Feb. 2003.

[6] I.V. Konoplev, A.J. MacLachlan, C.W. Robertson, et al., "Cylindrical, periodic surface lattice-Theory, dispersion analysis, and experiment," Appl. Phys. Lett., vol. 101, art. no. 121111, Sept. 2012.

[7] I. V. Konoplev, A. J. MacLachlan, C. W. Robertson, A. W. Cross and A. D. R. Phelps, "Cylindrical periodic surface lattice as a metadielectric: Concept of a surfacefield Cherenkov source of coherent radiation", Phys. Rev. A, vol. 84, art. no. 013826, July 2011.

[8] I. V. Konoplev, L. Fisher, A. W. Cross, A. D. R. Phelps, K. Ronald and C. W. Robertson, "Surface wave Cherenkov maser based on a periodic lattice", Appl. Phys. Lett., vol. 96 , art. no. 261101, June 2010.

[9] I .V. Konoplev, A. R. Phipps, A. D. R. Phelps, C. W. Robertson, K. Ronald, A. W. Cross, "Surface field excitation by an obliquely incident wave", Appl. Phys. Lett., vol. 102, art. no. 141106, Apr. 2013.

[10] I. Gibson , D. Rosen and B. Stucker, "Additive Manufacturing Technologies: 3D Printing, Rapid Prototyping, and Direct Digital Manufacturing", 2nd Edition, Springer, New York, USA, 2015..

[11] D. M. French, and D. Shiffler, "High power microwave source with a three dimensional printed metamaterial slow-wave structure," Rev. Sci. Instrum., vol. 87, art. no. 053308, May 2016.

[12] I.C. Somerville and S.J. MacGregor and I.O. Farish "An efficient stacked-Blumlein HV pulse-generator" Meas. Sci. and Tech., vol. 1, pp.866-868, Sep 1990 